\title{
Vacuum ultraviolet spectroscopy of the optical properties and electronic structure of seven poly(di-alkylsilanes)
}

\author{
R. H. French and J. S. Meth \\ Du Pont Co., Central Research, E356-323, Experimental Station, Wilmington, DE 19880 \\ (USA) \\ J. R. G. Thorne and R. M. Hochstrasser \\ University of Pennsylvania, Department of Chemistry, Philadelphia, PA 19104 (USA) \\ R. D. Miller \\ IBM Almaden Research Laboratory, 650 Harry Road, San Jose, CA 95120 (USA)
}

\begin{abstract}
We report the ultraviolet (UV) and vacuum ultraviolet (VUV) optical properties and electronic structure, up to $44 \mathrm{eV}$, of thin-film samples of seven poly(di-alkylsilanes) [alkyl =n-butyl, n-pentyl, i-hexyl, n-hexyl, n-octyl, and n-tetradecyl] with three types of Si-Si backbone conformations: helical, planar zigzag, and trans-gauche-trans-gauche' (TGTG'). The backbone conformation determines the UV transitions, with helical materials exhibiting one near-UV absorption, while two UV transitions are seen for the two-phase materials containing both the helical and planar zigzag backbone conformations. The TGTG' backbone exhibits a single UV absorption at $3.6 \mathrm{eV}$. At higher energies all materials show a prominent shoulder at $7.2 \mathrm{eV}$, with a doublet peak structure seen at $\sim 9$ and $\sim 12 \mathrm{eV}$. The $7.2 \mathrm{eV}$ transition is unaffected by the backbone conformation or alkyl substitution, while comparison with the electronic transitions in polyethylene shows that the high-energy double-peak structure corresponds to transitions in the hydrocarbon sidechains. The $\sim 12 \mathrm{eV}$ transitions appear to shift to higher energy with increasing sidechain length and in tetradecyl these sidechain transitions are noticeably narrowed, suggesting unusual sidechain crystallization in poly(di-n-tetradecylsilane). A hierarchy of electronic transitions can be developed whereby the UV transitions arise in the Si backbone, at intermediate energies backbone to sidechain transitions are observed, while the highenergy transitions are of the alkyl sidechains. This hierarchy in the electronic transitions demonstrates the ability of VUV spectroscopy of the electronic structure to serve as a microscopic probe of the bonding and structure of polysilanes, for example providing detailed insight into the properties of the sidechains in these polymers.
\end{abstract}

\section{Introduction}

Optical reflectance and transmission measurements, from the NIR to the vacuum UV and soft X-ray regions ( 0.5 to $44 \mathrm{eV}$ ), coupled with Kramers-Kronig (KK) analysis, determine the linear optical properties of a material. The complex dielectric constant, $\epsilon=\epsilon_{1}+i \epsilon_{2}$, encompasses the absorption and dispersion of the material due to electronic excitations arising from one- 
photon absorption processes. These measurements can provide a comprehensive understanding of the electronic structure and bonding [1].

The family of polysilane polymers is intriguing due to their diversity of chemical and spectroscopic properties and represents examples of silicon backbone polymers with low-dimensional $\sigma$-conjugated structures [2]. Of the di-alkyl polysilanes, di-n-hexylsilane (PDN6S) has received considerable attention due to its third-order optical nonlinearity, photoinduced birefringence, luminescence, and photolabile character. Changes in the alkyl group substitution, e.g., from butyl to tetradecyl, lead to a variety of room-temperature backbone conformations, including $7 / 3$ helical, planar zigzag and the trans-gauche-trans-gauche' (TGTG') types. This relationship between the alkyl sidechain and the backbone conformation, and the effects of polymer structure on the optical and electronic properties of the polysilanes, is of current interest.

Much of the work on the polysilanes has focused on understanding the first excited states of the conjugated $\mathrm{Si}-\mathrm{Si}$ backbone, which contributes strongly to the nonlinearity [3]. The one-photon allowed first excited states appear in the UV and have been studied previously [4]. In addition, the valence-band states of a polysilane have been studied using photoemission spectroscopy [5]. We have undertaken a broader study of the electronic structure utilizing optical spectroscopy from the visible to the vacuum ultraviolet (VUV) to determine the complete electronic structure and bonding of these polymers. From this we hope to elucidate how the electronic structure is related to the structural characteristics of the polymer, its backbone conformation, sidechains, and sidechain to backbone interactions, while also studying the conjugation and one-dimensional nature of these materials. We have focused on the poly(di-n-alkylsilanes), including polymers with n-butyl (PDN4S), n-pentyl (PDN5S), n-hexyl (PDN6S), n-heptyl (PDN7S), n-octyl (PDN8S) and n-tetradecyl (PDN14S) sidechains. In addition, we have studied poly(di-i-hexylsilane) (PDI6S), where the introduction of the isohexyl sidechain alters the backbone conformation from planar zigzag to a disordered helical conformation [6].

Electronic structure can be understood from either correlated electron (valence-bond) or one-electron theories. Correlated electron theories such as those described by Soos and Hayden [7] lead to the calculation of correlated 'many-electron' states. These models can explain the one- and two-photon transitions observed in the polysilanes [8]. From this perspective, higherlying electronic transitions might arise from a second allowed one-photon transition. Since Soos, in his correlated electron calculations using the Sandorfy model C [9], considered only two electrons per silicon, and neither the other $\mathrm{Si}$ valence electrons nor the valence electrons of the side chains were considered, these correlated electron models cannot supply a complete picture of the polymer's electronic structure. The use of the Sandorfy model H considers four electrons per silicon and can encompass the electronic structure of the backbone and sidechains [10]. These valence-bond calculations do not rely on long-range periodicity as is assumed in band models, and therefore 
can represent chains and the chromophoric segments that represent the chemically and structurally allowed conjugation lengths present in the polymer [11]. One-electron band theory, such as the $a b$ initio local density approximation (LDA) calculations of Mintmire [12], provides another perspective, which considers all of the valence electrons in the backbone and sidechains, but cannot explicitly deal with the effects of electron correlation, the formation of many-electron excitations such as excitons or the breakdown of longrange periodicity. The band-structure model of Mintmire and his calculated optical properties for helical and planar zigzag polysilanes show surprisingly good agreement with our measurements [1].

\section{Sample preparation}

The polysilane polymers studied were produced by a Wurtz-type coupling reaction of the respective silyl dichlorides and were purified by repeated reprecipitation to isolate the high-molecular-weight portion [13]. The polymers were dissolved in isooctane at dilutions up to 100:1 (w.v.), and the solutions spin-coated on to UV-grade fused silica substrates [14]. After spinning, the films were placed in vacuum ( $<1$ Torr) overnight to aid solvent evaporation, and were subsequently cooled for one hour using dry ice and flowing nitrogen. Cooling was used to ensure the phase transition to the room-temperature structure. The films ranged from $100 \mathrm{~nm}$ to $4 \mu \mathrm{m}$ in thickness. The thicker films were produced by spinning successive layers onto the substrate, without allowing the already deposited layers to dry completely. A sample of lowdensity polyethylene from a 4 mil bag was also measured to determine the transition energies of a simple hydrocarbon polymer with a composition comparable to the alkyl sidechains of the polysilane samples.

\section{Experimental}

Optical measurements were performed using two spectrophotometers. In the region from 0.5 to $6 \mathrm{eV}$, a Perkin Elmer Lambda $9 \mathrm{NIR} / \mathrm{Vis} / \mathrm{UV}$ spectrophotometer was used to measure the specular reflectance $(R)$ and transmission $(T)$ of the polymer film on a silica substrate. Specular reflectance measurements from 1.7 to $44 \mathrm{eV}$ were acquired using a unique VUV spectrophotometer with a laser plasma light source that has been previously described $[15,16]$. Determination of electronic structural information from optical reflectance measurements requires a Kramers-Kronig (KK) analysis [17] of the single-surface reflectance.

For thin-film samples on substrates, two additional contributions arise in the measured total reflectivity, i.e., the effects of the back surface and multiple internal reflections in regions where either the film or substrate are transparent and, for thin films, the effects of optical coherence, which give rise to interference fringes. The effect of multiple internal reflections is determined by the analysis of both the total $R$ and $T$ for a single film on 
substrate sample, to produce the single-surface $R$ and the absorption coefficient $\alpha$ [18]. For thin-film samples which show interference fringes, the analysis to remove the effects of interference is much more complex, since the solutions of the Fresnel equations become multivalued at certain wavelength and thickness combinations. We have recently made headway in solving this complex problem [19], and in this work samples that exhibited fringing were successfully analyzed using $R$ and $T$ measurements. The effects of optical coherence and fringe formation are not as severe in an optical density (OD) measurement, based solely on transmission measurements. However, these OD measurements have a much more limited dynamic range, and are unsuitable for determining the optical properties over the complete energy range of the electronic transitions. It is the capacity of the reflectance-based measurements to provide the complex optical properties, such as the index of refraction or the dielectric constant spanning the energy of all valence electron transitions, which makes reflectance the basis for comprehensive electronic structure measurements.

Upon determining the single-surface reflectance, $\mathrm{KK}$ analysis allows any of the complex optical properties to be calculated. We present our results in terms of the dielectric constant, where $\epsilon_{2}$ corresponds to the interband transitions between the valence and conduction bands (or the one-electron excitations between bonding and anti-bonding molecular orbitals), along with any excitonic transitions arising from one-photon excitation. The dielectric constant is a fundamental material parameter related to the electronic structure and bonding of the material, and its interpretation can be in terms of band structure, molecular orbital, or valence band models.

\section{Results}

The optical absorption coefficients of the poly(di-alkylsilanes) in the UV are shown in Fig. 1 and the energies of the UV transitions $\left(E_{1}\right.$ for helical and TGTG' samples and $E_{1}$ and $E_{1}{ }^{\prime}$ for samples containing predominantly the planar zigzag) are summarized in Table 1 along with the relevant backbone conformations. The samples were prepared using consistent procedures, yet variations in the optical spectra were observed for different materials. For example, PDI6S and PDN8S both exhibited a blue-shift of the UV transition energies for samples that were 100 to $150 \mathrm{~nm}$ thick as compared to samples greater than $300 \mathrm{~nm}$ thick; this may be a surface effect. The UV transition energies compare well with those of Schellenberg [20], who has also observed thickness-dependent variations in the $E_{1}$ and $E_{1}{ }^{\prime}$ energies. For the planar zigzag phases, the relative intensities of the $E_{1}$ and $E_{1}^{\prime}$ peaks varied from sample to sample; this may be due to the biphasic nature of these films, which contain varying amounts of the crystalline planar zigzag and residual disordered helical material in the sample.

The corrected single-surface reflectivities of the poly(di-alkylsilanes) are shown in Fig. 2. There is still some evidence of interference fringes in the 

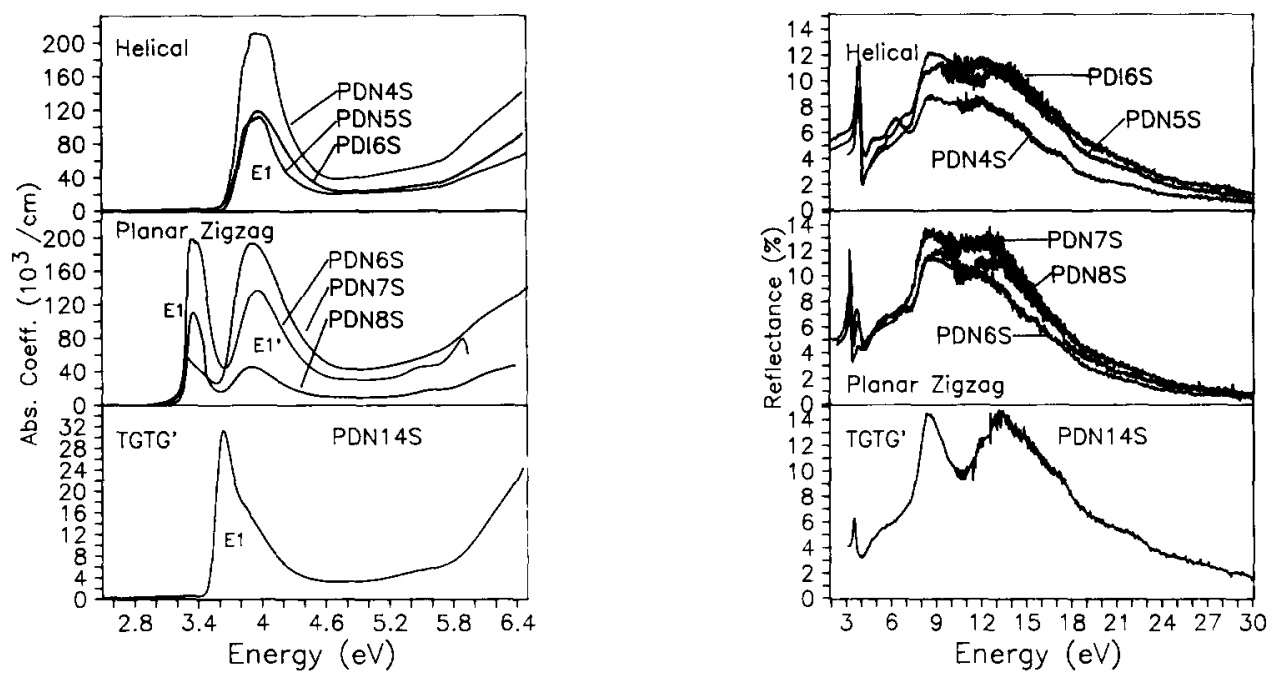

Fig. 1. (Left) Optical absorption of poly(di-alkylsilanes) PDN4S, PDN5S, PDI6S, PDN6S, PDN7S, PDN8S, and PDN14S, grouped by backbone conformation, showing the characteristic UV peaks of the $\mathrm{Si}-\mathrm{Si}$ backbone transitions.

Fig. 2. (Right) Single-surface optical reflectivity of poly(di-alkylsilanes) PDN4S, PDN5S, PDI6S, PDN6S, PDN7S, PDN8S, and PDN14S, grouped by backbone conformations, spanning the complete range of valence electronic transitions.

TABLE 1

Approximate transition energies (eV) of poly(di-alkylsilanes), determined from $\epsilon_{2}$

\begin{tabular}{lllllll}
\hline Polymer & $\begin{array}{l}\text { Structure } \\
\left(20{ }^{\circ} \mathrm{C}\right)\end{array}$ & $E_{1}$ & $E_{1}{ }^{\prime}$ & $E_{2}$ & $E_{3}$ & $E_{3}{ }^{\prime}$ \\
\hline PDN4S & $7 / 3$ helix $^{\mathrm{a}}$ & 4.00 & & 7.2 & 9.3 & 12.0 \\
PDN5S & $7 / 3$ helix $^{\mathrm{a}}$ & 3.98 & & 7.2 & 9.1 & 11.8 \\
PDI6S & Disordered helix $^{\mathrm{b}}$ & 4.04 & & 7.3 & 9.5 & 12.8 \\
PDN6S & Planar zigzagc $^{c}$ & 3.39 & 4.00 & 7.1 & 9.3 & \\
PDN7S & Planar zigzag $^{\mathrm{c}}$ & 3.31 & 3.98 & 7.1 & 9.3 & 12.5 \\
PDN8S & Planar zigzag $^{\mathrm{c}}$ & 3.38 & 3.94 & 7.1 & 9.2 & 12.1 \\
PDN14S & TGTG'c $_{\text {Polyethylene }}$ & 3.60 & & 7.2 & 8.8 & 12.5 \\
& & & & & 8.1 & 11.9
\end{tabular}

${ }^{2}$ F. C. Schillings, A. J. Lovinger, J. M. Zeigler, D. D. Davis and F. A. Bovey, Macromol., 22 (1989) 3055.

${ }^{b}$ Unpublished.

'R. D. Miller and J. Michl, Chem. Rev., 89 (1989) 1359.

reflectance of the PDN5S and PDI6S materials between the $E_{1}$ peak and the $E_{2}$ shoulder at $7.2 \mathrm{eV}$. In the other samples the effects of coherent interference have been adequately removed. The major features seen in the VUV are $E_{2}$ and $E_{3}$. In addition, the reflectivity of PDN14S is substantially sharper above $7.2 \mathrm{eV}$. The imaginary part of the dielectric constant $\left(\epsilon_{2}\right)$ determined from 
$\mathrm{KK}$ analysis is shown in Fig. 3. The energies of the $E_{2}$ and $E_{3}$ transitions have been tabulated in Table 1 . The highest-energy shoulder, $E_{3}{ }^{\prime}$, varied in amplitude from sample to sample in each material, and the energy is only tabulated in some cases. These energies have only been determined in a cursory manner. The appropriate approach for characterizing these electronic transitions is through the use of analytic critical point fitting of the real and imaginary parts of the dielectric constant, which properly determines the parameters of the interband transitions that give rise to the observed features [21]. The optical absorption coefficient for the complete range of electronic transitions is shown in Fig. 4, and demonstrates that the $E_{1}$ transitions, which range in absorption up to $2 \times 10^{5} \mathrm{~cm}^{-1}$, are dwarfed by the absorptions at higher energies, which reach values greater than $1 \times 10^{6} \mathrm{~cm}^{-1}$.

\section{Discussion}

The nature of the Si-Si bonding in the backbone and the $\mathrm{C}-\mathrm{C}$ bonding in the sidechains of the polysilanes leads to a natural separation of the electronic transitions of these polymers into three regions. At low energies, from 3 to $5 \mathrm{eV}, \sigma(\mathrm{Si}) \rightarrow \sigma^{*}(\mathrm{Si})$ transitions in the polymer backbone are seen. These transitions are sensitive to the backbone conformation. From 8 to 20 $\mathrm{eV}, \sigma($ alkyl $) \rightarrow \sigma^{*}$ (alkyl) transitions in the polymer sidechains occur, while at intermediate energies, from 5 to $8 \mathrm{eV}, \sigma\left(\right.$ Si-alkyl) $\rightarrow \sigma^{*}$ (Si-alkyl) transitions
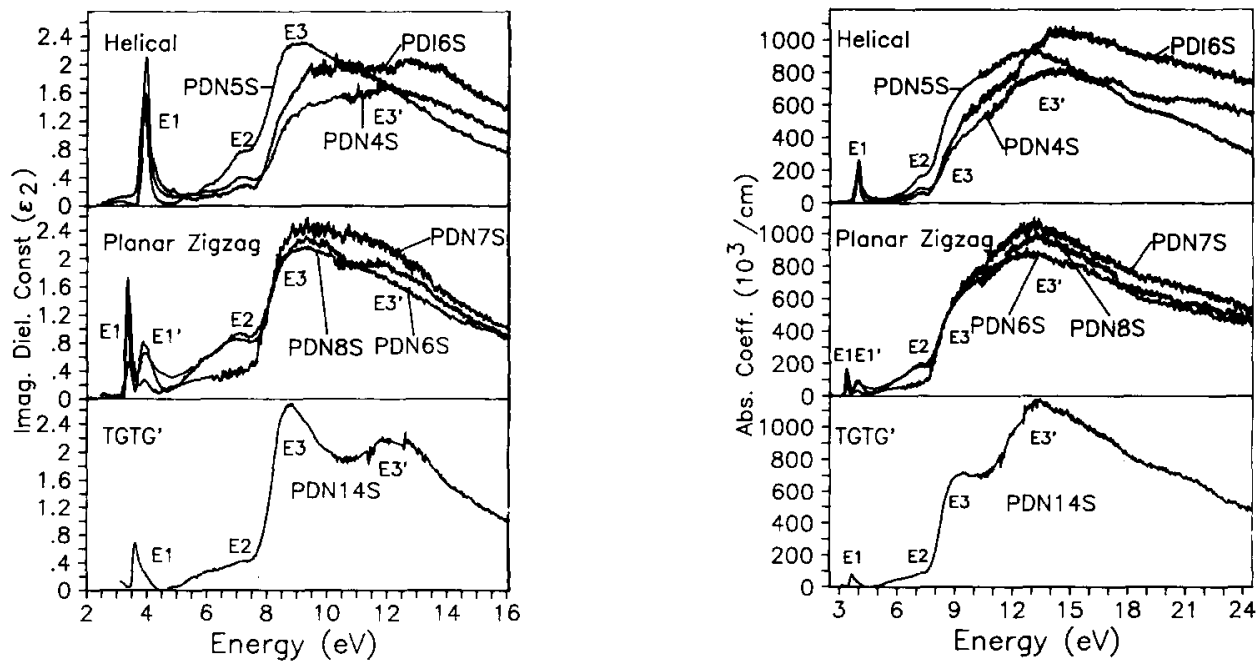

Fig. 3. (Left) Imaginary part $\left(\epsilon_{2}\right)$ of the complex dielectric constant of poly(di-alkylsilanes) PDN4S, PDN5S, PDI6S, PDN6S, PDN7S, PDN8S, and PDN14S, grouped by backbone conformation, showing the complete electronic structure.

Fig. 4. (Right) Optical absorption of poly(di-alkylsilane) PDN4S, PDN5S, PDI6S, PDN6S, PDN7S, PDN8S, and PDN14S, grouped by backbone conformation, in the energy range of the backbone and sidechain electronic structure transitions. 
arise between the backbone and the sidechain. These three spectral regions give us a microscopic probe of the bonding and structure of the polysilanes.

\subsection{Backbone $E_{1}$ transitions}

The most prominent example of this hierarchy of the electronic transitions is the interpretation of the $E_{1}$ peaks that are associated with the backbone conformation. For samples containing a planar zigzag backbone conformation, the lower energy of the $E_{1}$ peak is used as an indicator of this backbone conformation, while the $E_{1}{ }^{\prime}$ peak appearing at $\sim 4 \mathrm{eV}$, comparable to the $E_{1}$ energy seen in helical materials, represents the presence of another phase where the polymer backbone has a disordered helical conformation. For PDN14S a TGTG' backbone conformation results in a unique $E_{1}$ energy of $3.6 \mathrm{eV}$. This example shows that a red-shifted absorption relative to the helical forms cannot always be attributed to the presence of planar zigzag segments. This assignment of a single $E_{1}$ peak to each backbone conformation is further supported by the observation that, in predominantly planar zigzag materials, with sample history the $E_{1}$ and $E_{1}{ }^{\prime}$ peaks are seen to vary in amplitude inversely as the ratio of helical to planar zigzag backbone conformations in the sample varies.

\subsection{Sidechain $E_{3}$ transitions}

The energy of the $E_{3}$ peaks in the alkyl-substituted polysilanes corresponds closely to the observed transition energies of polyethylene reported in Table 1 and in the literature [22]. They also correspond reasonably with the reported VUV absorption energies for the $E_{3}$ peak of normal paraffin hydrocarbons [23] and perflouro-normal paraffins [24], even though the comparable $E_{3}$ transition energy in these gas-phase molecules was found to shift to lower energies with increasing carbon content. The $E_{3}$ peaks therefore give us insight into the structure and bonding in the alkyl sidechains. Just as the $E_{1}$ peaks can depend on the sample history, the $E_{3}{ }^{\prime}$ peak is seen to vary in amplitude from sample to sample, in Fig. 3 the amplitude of $E_{3}{ }^{\prime}$ in PDN5S and PDN6S is greatly reduced relative to the other poly(di-alkylsilanes). A particularly dramatic example of changes in the electronic structure of the sidechains is the sharpness and well-resolved nature of the $E_{3}$ peaks in PDN14S compared to the shorter-sidechain materials (Fig. 3). This decrease in the width of the $E_{3}$ sidechain transitions may arise from the mode of crystallization of the alkyl sidechains in PDN14S. One possibility is that in the tetradecyl derivative, the two sidechains from one silicon atom may interact together, instead of the interdigitating of the hexyl sidechains from adjacent polymer chains that apparently occurs in PDN6S [25]. Further speculation would be meaningless pending the complete structural elucidation of these polymers in the solid state. It would also be interesting to understand the cause of the $E_{3}{ }^{\prime}$ amplitude variations in the other polysilanes to determine if this gives further structural insight.

Considering $\epsilon_{2}$ (Fig. 3) as a function of n-alkyl sidechain length, while disregarding the differences in backbone conformation, the energy of the $E_{3}$ 
peak is almost unchanged with increasing number of carbons in the sidechain. The $E_{3}{ }^{\prime}$ transition, however, appears to shift to higher energy as the sidechain carbon number increases. This shift of $E_{3}{ }^{\prime}$ can be more easily seen in the absorption coefficient shown in Fig. 4. As mentioned above, for gas-phase molecules, the $E_{3}$ peak was found to shift to lower energies with increasing chain length, opposite to what is observed here for the polysilane alkyl sidechains. In X-ray and UV photoelectron studies of the valence bands of linear alkanes from $\mathrm{CH}_{2}$ to $n-\mathrm{C}_{13} \mathrm{H}_{28}$ [26] and of long-chain n-alkanes from $n-\mathrm{C}_{10} \mathrm{H}_{22}$ to $\mathrm{C}_{44} \mathrm{H}_{90}$ [27], the upper valence bands are found to have an approximate two-peak nature and to exhibit an increasing separation of these transitions with increasing carbon number, up to $\mathrm{C}_{13} \mathrm{H}_{28}$. For the longer alkane chains no shift is reported in the valence-band energies, suggesting that for the alkanes the electronic structure has converged to that of polyethylene. Confirmation of this shift of the $E_{3}{ }^{\prime}$ peak energy of the polysilane alkyl sidechains with increasing length awaits the analytical critical point analysis of these transitions.

A comparison of the $E_{3}$ transitions of isohexyl-substituted polysilane with the n-alkyl polysilanes shows that the $E_{3}{ }^{\prime}$ transition energy of the isohexyl sidechain is at noticeably higher energy, and does not follow the same trend as the $n$-alkyl sidechain transition energies. The change from an n-pentyl to n-hexyl sidechain results in a change in Si backbone conformation from helical to planar zigzag. The change in packing that occurs in going from the normal hexyl to the isohexyl sidechain not only shifts the $E_{3}{ }^{\prime}$ transition to higher energy but also results in a helical backbone conformation in the latter.

\subsection{Backbone $\rightarrow$ sidechain $E_{2}$ transitions}

The intermediate energy regime of electronic transitions of the polysilanes, characterized by the $E_{2}$ peak, is independent of changes in either the backbone conformation of the polymer or changes that arise from increasing sidechain length or sidechain crystallization. This transition is tentatively assigned to the backbone $\rightarrow$ sidechain excitations, which characterize all of these di-alkyl polysilanes. This transition apparently involves the $\mathrm{Si}-\mathrm{C}$ bond formed by the attachment of the hydrocarbon sidechain to the silicon backbone. Confirmation of this assignment awaits the study of polysilane derivatives with different types of substituents (e.g., aryl, alkoxy, etc.).

\section{Conclusions}

The optical properties and electronic structure of a family of seven poly(di-alkylsilanes), including n-butyl, n-pentyl, and i-hexyl, which have a helical backbone conformation, n-hexyl, n-heptyl, and n-octyl, which have planar zigzag backbone conformation, and n-tetradecyl, which has a TGTG' backbone conformation, have been studied. The $\mathrm{Si}-\mathrm{Si}$ backbone $E_{1}$ transitions arise from 3 to $5 \mathrm{eV}$ and are conformation dependent, as has been previously 
observed. At intermediate energies, from 5 to $8 \mathrm{eV}$, the $E_{2}$ transition at 7.2 $\mathrm{eV}$ is invariant with changes in the backbone conformation or alkyl sidechain substitution and is proposed to result at the point of attachment of the sidechain to the backbone. At higher energies still, the $E_{3}$ transitions arise from excitations isolated in the alkyl sidechains assigned by comparison to the spectra of polyethylene. The $E_{3}{ }^{\prime}$ transition appears to shift to higher energy as the carbon number of the n-alkyl sidechain increases. In addition, in the case of poly(di-n-tetradecylsilane), the $E_{3}$ transitions are dramatically sharper and more highly resolved. The complete electronic structure of the poly(di-alkylsilanes) as determined from vacuum ultraviolet spectroscopy supplies unique insight into the bonding and structure of these materials.

\section{Acknowledgements}

The authors would like to acknowledge the assistance of D. J. Jones and S. Loughin in the VUV spectroscopy.

\section{References}

1 F. M. Schellenberg, R. L. Byer, R. H. French and R. D. Miller, Phys. Rev. B, Rapid Commun., 43 (1990) 10008.

2 R. West, J. Organomet. Chem., 300 (1986) 327; R. D. Miller and J. Michl, Chem. Rev., 89 (1989) 1359; F. M. Schellenberg, R. L. Byer, R. D. Miller, R. H. French, S. S. Kano, Y. Takahashi, Y. Shiraki and R. Ito, in J. F. Harrod and R. M. Laine (eds.), Inorganic and Organometallic Oligomers and Polymers, Kluwer, Dordrecht, 1991, pp. 73-95.

3 J. R. G. Thorne, Y. Ohsako, J. M. Zeigler and R. M. Hochstrasser, Chem. Phys. Lett., 162 (1989) 6, 455; F. M. Schellenberg, R. L. Byer and R. D. Miller, Chem. Phys. Lett., 166 (1990) 331; J. R. G. Thorne, Y. Ohsako, S. T. Repinec, S. A. Abrash, J. M. Zeigler and R. M. Hochstrasser, J. Lumin., 45 (1990) 295; Z. G. Soos and G. W. Hayden, Chem. Phys., 143 (1990) 199; R. G. Kepler and Z. G. Soos, Phys. Rev. B, to be published; Y. Moritomo, Y. Tokura, H. Tachibana, Y. Kawabata and R. D. Miller, Phys. Rev. B, 43 (1991) 14746; H. Tachibana, Y. Kawabata, S. Koshihara and Y. Tokura, to be published.

4 P. Trefonas, R. West, R. D. Miller and D. Hofer, J. Polym. Phys.: Poly. Lett. Ed., 21 (1983) 823; J. F. Rabolt, D. Hofer, R. D. Miller and G. N. Fickes, Macromol., 19 (1986) 611; H. Kuzmany, J. F. Rabolt, B. L. Farmer and R. D. Miller, J. Chem. Phys., 85 (1986) 7413; L. A. Harrah and J. M. Zeigler, Macromol., 20 (1987) 601; V. M. Hallmark, R. Sooriyakumaran, R. D. Miller and J. F. Rabolt, J. Chem. Phys., 90 (1989) 2486; C. A. Walsh, F. C. Schilling, A. J. Lovinger, D. D. Davis, F. A. Bovey and J. M. Zeigler, Macromol., 23 (1990) 1742.

5 K. Seki, T. Mori, H. Inokuchi and K. Murano, Bull. Chem. Soc. Jpn., 61 (1988) 351.

6 K. Song, R. D. Miller, G. M. Wallraft and J. F. Rabolt, Macromol., 24 (1991) 4084-4088.

7 Z. G. Soos and G. W. Hayden, Chem. Phys., 143 (1990) 199.

8 Z. G. Soos and R. G. Kepler, Two-photon absorption spectrum of poly(di- $n$-hexylsilane) films, Phys. Rev. B, submitted for publication.

9 C. Sandorfy, Can. J. Chem., 33 (1955) 1337.

10 V. Balaji and J. Michl, Polyhedrom, 10 (1991) 11, 1265.

11 J. Michl, Symth. Met., 49-50 (1992) 367.

12 J. W. Mintmire, Phys. Rev. B, 39 (1989) 18, 13350.

13 P. Trefonas III, P. I. Djurovich, X. M. Zhang, R. West, R. D. Miller and D. Hofer, J. Polym. Sci. Polym. Lett. Ed., 21 (1983) 819. 
14 Dymasil 1000, Valpey Fisher, Hopkinton, MA 01748.

15 M. L. Bortz and R. H. French, Appl. Phys. Lett., 55 (1989) 19, 1955-1957.

16 R. H. French, Phys. Scr., 41 (1990) 4, 404-408.

17 M. L. Bortz and R. H. French, Appl. Spectrosc., 43 (1989) 8, 1498-1501.

18 M. E. Innocenzi, R. T. Swimm, M. Bass, R. H. French, A. B. Villaverde and M. R. Kokta, J. Appl. Phys., 67 (1990) 12, 7542-7546.

19 L. DeNoyer and R. H. French, unpublished work.

20 F. Schellenberg, Ph. D. Thesis, Applied Physics, Stanford University, 1991, Ginzton Laboratory Report No. 4850.

21 S. Loughin, R. H. French and L. DeNoyer, Critical point modeling of the interband transition strength of electrons, to be submitted for publication.

22 S. Hashimoto, K. Seki, N. Sato and H. Inokuchi, J. Chem. Phys., 76 (1982) 163; N. Ueno, K. Seki, K. Sugita and H. Inokuchi, Phys. Rev. B, 43 (1991) 2394.

23 B. A. Lombos, P. Sauvageau and C. Sandorfy, Chem. Phys. Lett., 1 (1967) 42.

24 G. Belanger, P. Sauvageau and C. Sandorfy, Chem. Phys. Lett., 3 (1969) 649.

25 P. Weber, D. Guillon, A. Skoulios and R. D. Miller, Liq. Cryst., 8 (1990) 6, 825.

26 J. J. Pireaux, S. Svensson, E. Basilier, P. A. Malqvist, U. Gelius, R. Caudano and K. Siegbahn, Phys. Rev. A, 14 (1976) 2133.

27 H. Ozaki and Y. Harada, J. Am. Chem. Soc., 112 (1990) 5735. 